\title{
Synovial Fluid Analysis - A Retrospective Study in a Tertiary Care Centre
}

IJCRR

Section: Healthcare

Sci. Journal Impact

Factor: 5.385 (2017)

ICV: 71.54 (2015)

\section{Karthikeyan $^{1}$, M. Sridevi ${ }^{2}$}

'MBBS, Saveetha Medical College and Hospital, Thandalam, Kancheepuram, Chennai - 602105; ${ }^{2}$ Associate Professor, Department of Pathology, Saveetha Medical College and Hospital, Kancheepuram, Chennai - 602105.

\section{ABSTRACT}

Introduction: Synovial fluid is a thick, stringy fluid found in the cavity of synovial joint.Its main function is to reduce the friction between two joints. Synovial fluid is made up of hyaluronic acid, lubricants and collagen. In a healthy joint the normal volume of synovial fluid is $0.15 \mathrm{mlto} 0.5 \mathrm{ml}$. This study was done with the objective to evaluate the characteristics of the synovial fluid in various joint diseases.

Method: The study was done in 60 cases of arthritis. Synovial fluid was collected and sent for physical and cytological analysis.

Result: Out of 60 cases, rheumatoid arthritis $(n=13 ; 19 \%)$ is the most common etiology followed by septic arthritis $(n=10 ; 17 \%)$. Male predominated over female. Inflammatory arthritis is predominant than non-inflammatory and septic arthritis in this study.

Conclusion: The synovial fluid analysis used for the investigation procedure in the diagnosis of various joint diseases. This study shows that rheumatoid arthritis is the most common cause for synovial inflammation.

Key Words: Arthritis, Joint diseases, Rheumatoid, Synovial fluid

\section{INTRODUCTION}

Synovial fluid analysis has been recommended as a routine procedure for the diagnosis of various joint diseases. Synovial fluid is normally found in bone joints. The main function of synovial fluid includes reduction of friction, shock absorption, nutrients and water transportation.

Arthritis can be either a monoarticular or polyarticular lesion leading to morbidity, affecting all ages and both sexes. Rheumatoid Arthritis is inflammatory arthritis which is the main cause for the inflammation of synovium. This inflammation results in joint pain and stiffness and if it progresses it will lead to joint damage and permanent loss of function ${ }^{1}$. Inflammation can be easily detected by doing a simple examination. At the time of presentation, mostly radiography shows normal finding but MRI and Ultrasound are highly sensitive to the smaller lesions and synovial inflammation ${ }^{2}$. Both synovial fluid analysis and synovial biopsy are advised routinely in cases of the joint diseases.
Ropes and Bauer was the first among to distinguish the appearance and cell content of abnormal synovial fluid and related to different disease categories, in particular, distinguishing inflammatory and non- inflammatory forms of arthritis ${ }^{3 .}$

\section{Inflammatory arthritis}

Inflammatory arthritis is characterized by inflammation of the joints and tissues. This includes Rheumatoid, Gout, and Tuberculous arthritis. Rheumatoid arthritis is an autoimmune disease where some research suggests that genetics plays an important role. Symptoms of inflammatory arthritis are pain, swelling, warmth and tenderness.

\section{Non- inflammatory arthritis}

Even though it is called non-inflammatory arthritis, it can still result in some inflammation of the joint. This includes osteoarthritis, Haemarthrosis etc. It is mainly due to the breakdown of joint cartilage and it is not an autoimmune disease.

\section{Corresponding Author:}

Dr. M. Sridevi, Department of Pathology, Saveetha Medical College and Hospital, Thandalam, Kancheepuram, Chennai - 602105. Phone: 9841251860; Email: dr.sridevi81@gmail.com

ISSN: 2231-2196 (Print)

Received: 08.07 .2019
ISSN: 0975-5241 (Online)

Revised: 12.08 .2019
Accepted: 23.08 .2019 


\section{MATERIAL AND METHODS}

This study was done in Saveetha Medical College, Thandalam in 60 patients having joint pathogenesis visiting our Outpatient department. The period of study was from July 2018 to December 2018. The patients were completely examined and a clear history was taken. Symptoms like morning stiffness, frequency and periodicity of the episode with characteristic waxing and anorexia, asthenia, cough were important constitutional enquiries made in cases where systemic diseases were suspected. A special effort was made to probe and find out any history suggestive of non-inflammatory osteoarthritis, chronic infection and inflammation like tuberculosis, rheumatoid arthritis and gout and severe inflammation like septic arthritis.

After complete examination they are subjected to synovial fluid analysis and in some synovial biopsy. Before doing the biopsy a small amount of fluid were collected for the analysis and the fluid was sent for the:

- Physical analysis

- Biochemical analysis

- Clinical pathology and Cytology

The physical analysis includes color, appearance and volume. Biochemical analysis includes glucose levels and protein levels in synovial fluid. Cytology includes the total leukocyte count and differential count from the centrifuged deposit to see the predominant white blood cells(WBCs).

\section{Arthrocentesis}

Arthrocentesis is a surgical procedure done to aspirate the synovial fluid from the affected joints. Full aseptic surgical care was taken in the local preparation, the draping of the patient and scrubbing by the surgeon. About $2 \mathrm{ml}$ of $2 \%$ lignocaine was injected into the skin, subcutaneous tissue and joint capsule. The knee was kept in extension; the synovial fluid was displaced from supra patella pouch to the medial or lateral aspect of the patella. After infiltration of the joint capsule, the needle was introduced through a point on lateral aspect $2 \mathrm{~cm}$ above and $2 \mathrm{~cm}$ lateral to the midpoint of the upper border of patella into the joint cavity and synovial fluid aspirated and collected in test tubes ${ }^{[4]}$.

The needle was taken out leaving the cannula. A $20 \mathrm{ml}$ of syringe has already been fitted with a notched needle is inserted through the mouth of the cannula so that the blunt end of the needle can easily enters the synovial cavity. Strong suction was then applied to the barrel and few $\mathrm{ml}$ of synovial fluid is collected. The suction was maintained to hold the synovial specimen within the notch. The syringe and the inner needle were held motionless in the right hand where left hand slowly advanced the outer cannula using a slight twisting and rotating motion for about $1 \mathrm{~cm}$ to ensure that specimen has been severed and held in the notch. After aspiration, the needle is removed by leaving the cannula behind. ${ }^{4}$ Using another needle a piece of tissue is taken and then transferred it to the formalin and sent for histopathology analysis.

\section{RESULTS}

This study was performed in Saveetha Medical College. The total number of cases chosen for the study is 60 of which 51 cases were specific arthritis whereas 9 cases were non- specific arthritis.

Table1 shows the age pattern in different joint diseases. According to our study Rheumatoid arthritis was found between the age group of 31-50 and above 50 predominantly. Tubercular arthritis was found mainly in the younger age group between 11-30 years. Septic arthritis, Osteoarthritis and Gout were more common in age group between 31-50 years.

Sex distribution of diseases as given in Table 2, shows male predominant over femalein this study whereas in haemarthrosis females were more affected than male.

Number of cases and their percentagesaregiven in Table 3. Our study indicates that rheumatoid arthritis (19\%) and septic arthritis (17\%) are more common than Osteoarthritis, tuberculosis arthritis and gout. Traumatic arthritis, haemarthrosis and other non- specific arthritis were least common in our study.

Physical properties of synovial fluid in different diseases showed turbid appearance except for gout in which it showed clear appearance. Amount of volume varied from $3 \mathrm{ml}$ to $30 \mathrm{ml}$. Pale yellow and yellowish colour was the most predominant colour of the synovial fluid in most of the diseases whereas reddish colour was seen in cases of haemarthrosis, traumatic and rheumatoid arthritis.

Cytological study of synovial fluid shows differential cell count and predominant cell in disease condition in Table 4. There is increased count of WBC cells in rheumatoid arthritis (7000-15000) and gout (7000-13500 cells/ cu.mm). Polymorphic cells were more predominantly seen in rheumatoid arthritis, traumatic arthritis, Septic arthritis, osteoarthritis, whereas lymphocytes were predominantly seen in tuberculous arthritis and haemarthrosis.

\section{DISCUSSION}

Synovial fluid analysis has been routinely recommended for the evaluation of arthritis with joint effusions. ${ }^{[5]}$ Robes and Bauer were the first to categorize and distinguish inflammatory from non- inflammatory forms of arthritis based on the appearance and cell content of the synovial fluid ${ }^{3}$. Hollander and McCarty were the first to introduce polarized light microscopy of synovial fluid to identify Urate and Pyrophos- 
phate crystals in the definitive diagnosis of gout and pseudo gout. ${ }^{6}$

According to the study done by Ganesh et al the most commonly affected age group was 31-50 years which coincided with the present study. Similarly the total WBC count and the predominant cell type present in the affected joint is almost tallied with the study done by Ganesh et al ${ }^{4}$

Based on the synovial fluid evaluation, the various types of arthritis were grouped and compared with the findings of previous studies. In the present study, Rheumatoid Arthritis $(13$ cases $)$ was the most common arthritis next to septic arthritis (10 cases) and the next common is osteoarthritis (8 cases), tuberculosis arthritis (8 cases) and gout (7 cases).

Rheumatoid arthritis (RA) is a painful inflammatory arthritis characterized by symmetrical inflammation of the synovium of the small joints of the hands, wrist and feet leading to progressive joint damage like deformities and loss of function. ${ }^{4}$

Hematogenous infection and direct inoculation of joint during surgery or trauma are the significant causes of Septic arthritis. It is more prevalent among the pediatric age population. $^{7}$

Gout is a systemic disease which results from hyperuricemia with deposition of needle shaped monosodium urate crystals in the tissues (Figure no 1), particularly joints which can be confirmed using Polarized light microscopy. ${ }^{8}$

In our study the most common condition wasRheumatoid arthritis, which is in concordance withthe study done by Venkatraman et al and Singhal et al $1^{9,10}$ where the prevalent cause was Tuberculous arthritis. Chronic non- specific synovitis was used in the absence of diagnostic features of specific inflammation.

Closed needle biopsy is a simple outpatient procedure without complications that aids in establishing the diagnosis after clinical and radiological correlation. This type of simple procedure plays a vital role in differential diagnosis of joint diseases which has been studied extensively by various authors $^{11,12}$

Further, it has been mentioned in the literature that the macroscopic features of inflammation seen at arthroscopy do not predict the microscopic features. Thus, the use of closed needle biopsy technique is justified. ${ }^{13}$

\section{CONCLUSION}

Synovial fluid analysis will give us an idea about the differential diagnosis of joint diseases. Synovial fluid aspiration should be done for the analysis and also used as a treatment procedure of synovial inflammation. Cytology reveals the predominant cells involved in the inflammatory disorders and also the normal content of the synovial fluid.

Funding: No funding sources

Limitations: None

Conflicts of interest: No potential conflict of interest relevant to this article was reported

Ethical approval: Approved.

\section{ACKNOWLEDGEMENT}

Authors acknowledge the immense help received from the scholars whose articles are cited and included in references of this manuscript. The authors are also grateful to authors / editors / publishers of all those articles, journals and books from where the literature for this article has been reviewed and discussed.

\section{REFERENCE}

1. Kahlenberg JM, Fox DA. Advances in the medical treatment of rheumatoid arthritis. Hand Clin.2011; 27(1):11-20.

2. Vosse D, de Vlam K. Osteoporosis in rheumatoid arthritis and ankylosing spondylitis. Clinical and experimental rheumatology.2009; 27:62.

3. Ropes MW, Bauer W. Synovial Fluid Changes in Joint Disease. Cambridge, Mass: Harvard University Press; 1953.

4. Ganesh K Reddy, Rallapalli R, Galla SS, Ravindran B. A prospective study for diagnosing joint diseases by synovial fluid analysis and percutaneous needle biopsy of synovium. Int J Res Orthop2017;3:661-9

5. S Abdullah, S A Young-Min, S J Hudson, C A Kelly, C R Heycock, J D Hamilton. Gross synovial fluid analysis in the differential diagnosis of Joint effusion. J ClinPathol 2007; 60:1144 1147.

6. Hollender JL. Examination of synovial fluid as a diagnostic aid in arthritis. Med Clin North Am, 1966, 50:1281-93.

7. Vijay PM, Doddikoppad MM. Clinicopathological study of inflammatory synovial lesion. Int J Biol Med.2011; 2(4):882-8.

8. Gaafar Ragab, Mohsen Elshahaly, Thomas Bardin. Gout: An old disease in new perspective - A review. Journal of Advanced Research 8 (2017) 495-511.

9. Singhal O, KaurV, Kalhan S, Singhal MK, Gupta A, Machave YV. Arthroscopic synovial biopsy in definitive diagnosis of joint diseases: An evaluation of efficacy and precision. Int J App Basic Med Res.2012;2:102-6

10. Venkataraman M., Sathyadharan P. Role of Needle Synovial Biopsy in Joint Diseases. J Evol Med Dental Sci.2015; 4(44):762634.

11. Verma R, Gupta SP, Vinod K. Place of synovial fluid examination and needle biopsy of synovial membrane in diagnosis of monoarticular joint diseases. Ind J Ortho.1983; 17(1):8-12.

12. Sakhuja A, Singh S, Chaturvedi S, Bajpai J, Shukla R. Synovial fluid analysis and synovial biopsy in various types of arthritis's. Indian J Orthopaed. 1981; 15(2):157.

13. Bresnihan B. Synovial biopsy in arthritis research: five years of concerted European collaboration. Ann Rheumatic Dis.2000; 59(7):506-11. 
Table 1: Showing age distribution pattern in different joint diseases.

\begin{tabular}{lcccccc} 
Name of the disease & $\begin{array}{c}\text { Number of } \\
\text { cases }\end{array}$ & 0-10 & $\mathbf{1 1 - 3 0}$ & $\mathbf{3 1 - 5 0}$ & $\mathbf{5 1 - 6 0}$ & Above 60 \\
Rheumatoid arthritis & 13 & 1 & 3 & 4 & 3 & 2 \\
Tuberculous arthritis & 8 & - & 3 & 2 & 2 & 1 \\
Gout & 7 & - & 1 & 4 & 2 & - \\
Traumatic arthritis & 3 & - & 1 & 1 & 1 & - \\
Haemarthrosis & 2 & - & 2 & - & - & - \\
Septic arthritis & 10 & - & 2 & 6 & 1 & 1 \\
Osteoarthritis & 8 & - & 2 & 3 & 1 & 4 \\
Non specific arthritis & 9 & - & 1 & 2 & 5 & 1 \\
\hline
\end{tabular}

Table 2: Sex distribution of diseases

\begin{tabular}{lcc} 
Diseases & Male & Female \\
Rheumatoid diseases & 9 & 4 \\
Tuberculous arthritis & 5 & 3 \\
Gout & 7 & - \\
Traumatic arthritis & 1 & 2 \\
Haemarthrosis & - & 2 \\
Septic arthritis & 8 & 2 \\
Osteoarthritis & 6 & 2 \\
Nonspecific synovitis & 9 & - \\
\hline
\end{tabular}

Table 3: Showing number of cases and its percentage

\begin{tabular}{lcc} 
Name of the diseases & Number of cases & Percentage \\
Rheumatoid diseases & 13 & $19 \%$ \\
Tuberculous arthritis & 8 & $13 \%$ \\
Gout & 7 & $12 \%$ \\
Traumatic arthritis & 3 & $5 \%$ \\
Haemarthrosis & 2 & $3 \%$ \\
Septic arthritis & 10 & $17 \%$ \\
Osteoarthritis & 8 & $13 \%$ \\
Nonspecific synovitis & 9 & $15 \%$ \\
\hline
\end{tabular}


Table 4: Cytological appearance of diseased joint

\begin{tabular}{lll} 
Diseases & Total WBC count & Predominant cells \\
Rheumatoid diseases & $7000-15000$ & Neutrophils- $60-100 \%$ \\
Tuberculous arthritis & $500-7000$ & Lymphocytes $=70-90 \%$ \\
Gout & $7000-13500$ & $\begin{array}{l}\text { Lymphocytes }=17-98 \% \\
\text { Polymorphic }=80 \%\end{array}$ \\
Traumatic arthritis & $100-6000$ & Neutrophils $=100 \%$ \\
Haemarthrosis & $1000-8000$ & Lymphocytes $=30-98 \%$ \\
septic arthritis & $1000-7000$ & Neutrophils $=95 \%$ \\
Osteoarthritis & $200-500$ & Neutrophils $=50-95 \%$ \\
Nonspecific synovitis & $400-10000$ & Neutrophils $=40-99 \%$ \\
& & Macrophages $=40 \%$ \\
& & Histiocytes $=10 \%$ \\
\hline
\end{tabular}

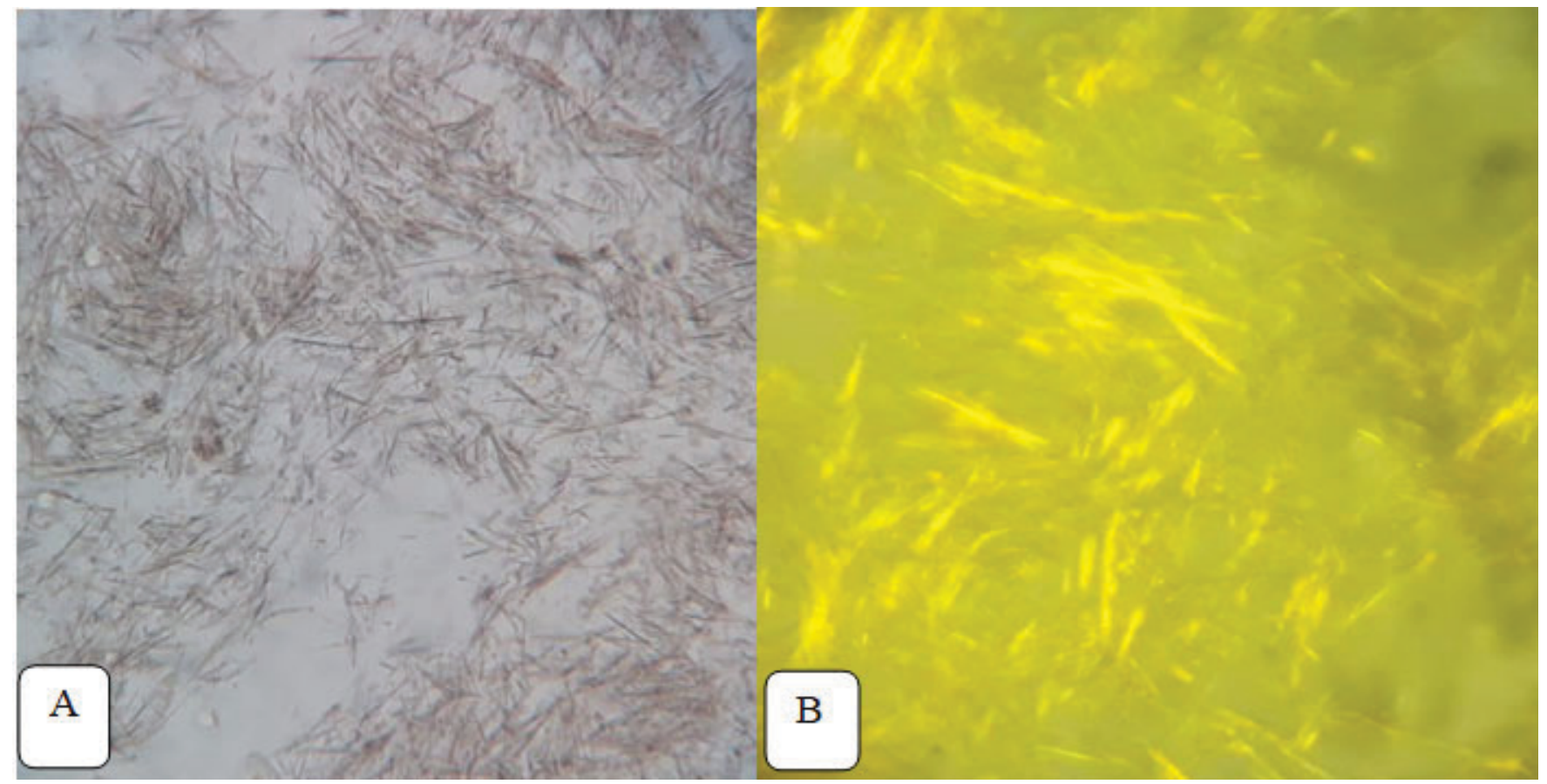

Figure 1: The light microscopicimage (A) and the polarized microscopic image (B) of gout shows the needle shaped monosodium Urate crystals. 